\title{
Vancomycin-resistant enterococcal infection in a Thai university hospital: clinical characteristics, treatment outcomes, and synergistic effect
}

This article was published in the following Dove Press journal: Infection and Drug Resistance

\author{
Jatapat Hemapanpairoa ${ }^{1-3}$ \\ Dhitiwat Changpradub ${ }^{4}$ \\ Sudaluck Thunyaharn ${ }^{5}$ \\ Wichai Santimaleeworagun ${ }^{3,6}$ \\ 'Department of Pharmacy Practice and \\ Pharmaceutical Care, Faculty of \\ Pharmaceutical Sciences, Burapha \\ University, Chonburi 20131, Thailand; \\ ${ }^{2}$ College of Pharmacotherapy Thailand, \\ Nonthaburi II000, Thailand; \\ ${ }^{3}$ Pharmaceutical Initiative for Resistant \\ Bacteria and Infectious Diseases Working \\ Group, Nakorn Pathom 73000, Thailand; \\ ${ }^{4}$ Division of Infectious Disease, \\ Department of Medicine, \\ Phramongkutklao Hospital, Bangkok \\ 10400, Thailand; ${ }^{5}$ Faculty of Medical \\ Technology, Nakhonratchasima College, \\ Nakhon Ratchasima 30000, Thailand; \\ ${ }^{6}$ Department of Pharmacy, Faculty of \\ Pharmacy, Silpakorn University, Nakorn \\ Pathom 73000, Thailand
}

\begin{abstract}
Purpose: The incidence of infections with vancomycin-resistant enterococci (VRE) is increasing, with associated high mortality rates and limited therapeutic choices. We investigated the clinical characteristics and treatment outcomes of VRE infection and also determined the in vitro effect of monotherapy and combined antimicrobials against clinical VRE isolates.
\end{abstract}

Patients and methods: Clinical data and bacterial isolates obtained from patients with VRE infections between January 2014 and April 2018 at Phramongkutklao Hospital were reviewed. The clinical outcomes included in-hospital mortality, 30-day mortality, and microbiological eradication. Clonal relationships were assessed by random amplified polymorphic DNA analysis. In vitro activity of linezolid, tigecycline, fosfomycin, gentamicin, chloramphenicol, and ampicillin were determined by minimum inhibitory concentration (MIC) values. Tests of synergy of fosfomycin- or gentamicin-based combinations by the checkerboard method were reported with the fractional inhibitory concentration index or MIC reduction, respectively.

Results: Among 26 cases of VRE infection, nosocomial and gastrointestinal infections were the most common. There were various treatment regimens, but linezolid-containing regimens were generally used. In-hospital and 30-day mortality were $73.1 \%$ and $57.7 \%$, respectively. Higher mortality was significantly associated with illness severity. The VRE isolates tested were universally susceptible to linezolid and tigecycline. A synergistic or additive effect was observed for fosfomycin combined with linezolid (100\%) and with tigecycline $(83.3 \%)$. Fourfold or greater MIC reduction was observed for linezolid or fosfomycin plus gentamicin at concentrations $1(58.3 \%, 62.5 \%), 2(83.3 \%, 62.5 \%)$, and $4 \mu \mathrm{g} / \mathrm{mL}(91.6 \%, 62.5 \%)$.

Conclusion: In-hospital mortality among patients with VRE infection was high. Linezolid remains a treatment of choice. However, combination therapy such as linezolid plus fosfomycin and linezolid plus gentamicin should be considered in cases of serious infection.

Keywords: clinical outcomes, vancomycin-resistant enterococci, minimum inhibitory concentration, synergism

\section{Introduction}

Although enterococci are part of the normal flora and have low virulence, they can cause serious infection in debilitated patients. Enterococcus faecalis and Enterococcus faecium are major causes underlying enterococcal infections in humans, such as bacteremia, endocarditis, intra-abdominal and pelvic infection, and urinary tract infection. Enterococci are intrinsically resistant to many antibiotics. The treatments of choice for ampicillin-susceptible and -resistant enterococci are ampicillin and vancomycin, respectively. However, inappropriate vancomycin use
Correspondence: Wichai

Santimaleeworagun

Department of Pharmacy, Silpakorn

University, RajamankhaNai Road, Nakhon

Phathom 73000, Thailand

Tel +66 34255800

Fax +6634255801

Email swichail234@gmail.com 
has resulted in the emergence of vancomycin-resistant enterococci (VRE), especially E. faecium. Enterococci can spread resistance genes to other organisms. ${ }^{1}$ Patients can acquire VRE infections from the hospital environment or because of colonization with VRE. ${ }^{1,2}$

Invasive VRE infection is difficult to treat and is associated with a high mortality rate. ${ }^{3}$ Compared with vancomycin-sensitive enterococcal bacteremia, VRE bacteremia is associated with a higher mortality rate, longer length of hospital stay, and higher treatment costs. ${ }^{3,4}$ Delay in administering appropriate antibiotic therapy is associated with increased mortality. World Health Organization states that vancomycin-resistant E. faecium is a "high priority pathogen" for which new antibiotics are urgently required. ${ }^{5}$ The antibiotics currently available to treat VRE include linezolid, daptomycin, quinaprintin/dalfopristin, teicoplanin, tigecycline, fosfomycin, and other newer agents such as tedizolid, oritavancin, and dalbavancin. Of these, only linezolid, tigecycline, teicoplanin, and fosfomycin are available in Thailand. Treatment choices are based on adverse effects, antibiotic penetration, and drug interactions. However, single antibiotic regimens are often only bacteriostatic against enterococci and inadequate in several cases of serious infection. ${ }^{2}$ Thus, the focus is currently on antibiotic combinations to treat VRE infections. ${ }^{6}$

The incidence of nosocomial VRE infections is increasing in several countries, including Thailand. According to the National Antimicrobial Resistance Surveillance Center, Thailand, vancomycin resistance rates in E. faecium strains increased from $0.7 \%$ in 2012 to $8 \%$ in 2018 . However, resistance rates in E. faecalis strains remained stable over a 10 -year period $(0.4 \%$ in 2018). ${ }^{7}$ However, evidence on treatment patterns and clinical outcome in Thailand are lacking. There was only one study evaluating antimicrobial activity against VRE over the decade 1999-2009, but it did not include treatment regimens or outcomes of VRE infections. ${ }^{8}$ In the present study, we aimed to evaluate the in vitro susceptibility of clinical enterococcal isolates to single and combined antibiotics and to determine treatment outcomes in Thai patients infected with VRE.

\section{Materials and methods}

\section{Patients and clinical strains}

We retrospectively reviewed clinical data and bacterial isolates of adult patients in whom VRE infection was detected between January 2014 and April 2018 at
Phramongkutklao Hospital, a 1,200-bed university hospital in Bangkok, Thailand. VRE infection criteria were based on the Centers for Disease Control and Prevention/ National Healthcare Safety Network Surveillance Definitions for Specific Types of Infections. ${ }^{9}$ Patients who were unavailable for follow-up of treatment outcome or who received $<3$ days of antibiotics were excluded.

\section{Ethical approval}

This study was approved by the ethics review committee of the Royal Thai Army Medical Department, Bangkok, Thailand (approval no. Q017b/61). This study was performed in accordance with the Declaration of Helsinki. We collected data after obtaining ethical approval and permission from the Director of the Phramongkutklao Hospital. Patient's personal data were collected and analyzed in anonymity and confidentially. Identifying information of participants was not collected and only the researcher can access the data. Patient informed consent was not required by the ethics review committee of the Royal Thai Army Medical Department because the ethics review committee often permit a waiver of consent for using archived bacterial isolates, retrospective chart review studies, confidential and anonymized data.

\section{Clinical characteristics and treatment outcomes}

Patient data were obtained from electronic medical records. Information collected included demographic data, comorbidities, neutropenia if present, admission dates, admitting ward, antimicrobial use, infection dates, specimen for microbial culture, isolates, types of infections, severity of illness, exposure to a mechanical ventilator or urinary catheter, antimicrobial susceptibility, definitive treatment of VRE infection, days until definitive antibiotic treatment, and outcome of treatment. The primary study outcome was in-hospital all-cause mortality, and secondary outcomes were 30-day all-cause mortality and microbiological cure after 3 days of definitive antibiotics.

\section{Molecular typing}

Genomic DNA was extracted from clinical bacterial isolates using a commercial kit (Thermo Fisher Scientific, Waltham, MA, USA). Clonal typing was performed using RAPD with ERIC1R (5'-ATG TAA GCT CCT GGG GAT TCA C-3') and AP4 (5'-TCA CGC TGC A-3') primers according to Barbier et al. ${ }^{10}$ Polymerase chain reaction amplification was 
performed in a Biometra TGradient Thermocycler (Biometra, Gottingen, Germany) at $94^{\circ} \mathrm{C}$ for $3 \mathrm{mins}$ followed by 44 cycles at $94^{\circ} \mathrm{C}$ for $30 \mathrm{~s}, 35^{\circ} \mathrm{C}$ for $1 \mathrm{~min}$, and $72^{\circ} \mathrm{C}$ for 2 mins. Ramping transition was $90 \mathrm{~s}$ from $65^{\circ} \mathrm{C}$ to $35^{\circ} \mathrm{C}$ and $111 \mathrm{~s}$ from $35^{\circ} \mathrm{C}$ to $72^{\circ} \mathrm{C}$. RAPD products were run on $1.5 \%$ agarose gels and stained with ethidium bromide (Bio Basic, Markham, Ontario, Canada). RAPD patterns were classified in groups. ${ }^{11}$

\section{In vitro activity of single and combined antimicrobial agents}

The minimum inhibitory concentration (MIC) values of antibiotics were evaluated in vitro for each clinical VRE strain. Linezolid, tigecycline, and fosfomycin MICs were determined by E-test methods (Liofilchem, Teramo, Italy). Ampicillin, chloramphenicol, gentamicin, and vancomycin MICs were evaluated by broth microdilution (standard powder donated by the Siam Pharmaceutical Co., Ltd.) Testing conditions were at $35-37^{\circ} \mathrm{C}$ in ambient air for 16-18 hrs, except for vancomycin (tested for $24 \mathrm{hrs}$ ) according to the Clinical and Laboratory Standards Institute (CLSI) recommendations. ${ }^{10}$

Susceptibility categories of linezolid, ampicillin, chloramphenicol, vancomycin, fosfomycin, and gentamicin were reported using CLSI criteria. Susceptibility category of tigecycline was based on the European Committee on Antimicrobial Susceptibility Testing criteria. ${ }^{12,13}$ Susceptibility categories of ciprofloxacin and levofloxacin were based on laboratory test results.

A combination of microdilution and checkerboard testing was used to determine synergistic activity of anti-VRE drugs. The VRE strains for which the MIC of fosfomycin was $>1,024 \mu \mathrm{g} / \mathrm{mL}$ and strains that were highly resistant to gentamicin (MIC>500 $\mu \mathrm{g} / \mathrm{mL}$ ) were excluded from combination testing. The checkerboard assay was performed in 96-well microtiter panels with concentrations of linezolid of $0.125-8 \mu \mathrm{g} / \mathrm{mL}$, tigecycline $0.007-8 \mu \mathrm{g} / \mathrm{mL}$, fosfomycin $8-512 \mu \mathrm{g} / \mathrm{mL}$, and gentamicin $0.25-4 \mu \mathrm{g} / \mathrm{mL}$.

Synergistic activity of fosfomycin combined with linezolid or tigecycline was determined by the fractional inhibitory concentrations (FIC) index. Synergistic, additive, indifferent, and antagonistic effects were defined by an FIC index of $\leq 0.5,>0.5-1,>1-4$, and $>4$, respectively. The effect of gentamicin combined with fosfomycin or with linezolid was determined by assessing the reduction in the MIC by the combination with gentamicin compared with the MICs of fosfomycin and linezolid alone.

\section{Definitions}

Comorbidities were assessed by the Charlson Co-morbidity Index (CCI) and the severity of illness by the Simplified Acute Physiology Score (SAPS II) on the day positive cultures were reported. ${ }^{14,15}$ Antimicrobial use was defined as an antibiotic administered for at least 3 days within 30 days prior to VRE infection. Neutropenia was defined as a neutrophil count $<500 / \mathrm{mm}^{3}$ within 30 days prior to VRE infection. Polymicrobial infection was defined as the isolation of other pathogens within $24 \mathrm{hrs}$ from the same site from which VRE were isolated. Days to definitive antibiotics were defined as the number of days before antibiotics appropriate for VRE were administered. Appropriate treatment was defined as an antibiotic regimen, either monotherapy or a combination, that matched the bacterial susceptibility data from this study and the hospital laboratory standards.

\section{Statistical analysis}

We reported descriptive statistics for clinical characteristics, susceptibility patterns, and the MICs by 50th percentile (MIC50) and 90th percentile (MIC90). The chi-square test and the Mann-Whitney U test were used to compare categorical and continuous variables between groups. Multivariate analyses by logistic regression were used to explore factors associated with higher mortality. Statistical significance was accepted when $P<0.05$. Analysis was performed with $\mathrm{R}$ program (version 3.5.2).

\section{Results}

From January 2014 to April 2018, among 39 adults with VRE infection, 12 received antibiotics for $<3$ days and 1 was referred to another hospital. This left 26 patients with VRE infections whose records were included in the study. All infections were with E. faecium.

\section{Clinical characteristics and treatment outcomes}

Of the 26 patients (Tables 1 and 2) with VRE infections, $19(73.1 \%)$ were men, and the median age was 68.5 years (IQR 57-80 years). The median SAPII and CCI were 53 (IQR 34-61) and 4.5 (IQR 2-6), respectively. All patients were hospitalized for more than 48 hrs (range, 6 days to 5 months). Prior to the VRE infection, 14 (53.8\%) patients had been admitted to the intensive care unit, $16(61.5 \%)$ required mechanical ventilation, and 15 (57.7\%) had an indwelling urinary catheter. Carbapenems and vancomycin 


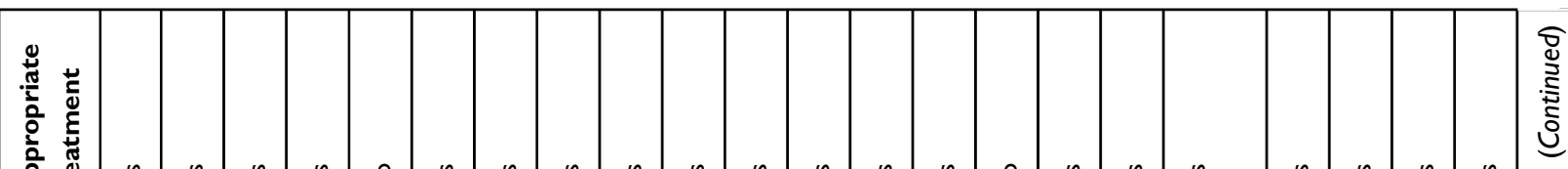

帝 站

\begin{tabular}{|c|c|c|c|c|c|c|c|c|c|c|c|c|c|c|c|c|c|c|c|c|c|c|}
\hline 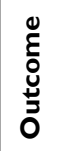 & 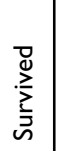 & סֶ, & 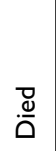 & $\stackrel{\square}{\square}$ & $\stackrel{\square}{\square}$ & סֶ, & 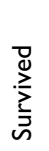 & 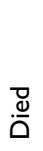 & 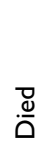 & $\stackrel{\square}{\frac{\tilde{\omega}}{\Delta}}$ & 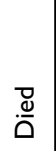 & $\stackrel{\square}{\square}$ & $\stackrel{\square}{\square}$ & 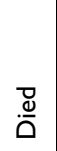 & סֶ, & $\stackrel{\square}{\square}$ & סֶ, & 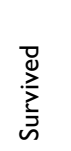 & & $\stackrel{\square}{\square}$ & 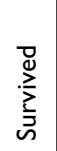 & 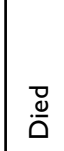 \\
\hline
\end{tabular}

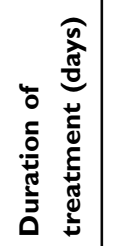

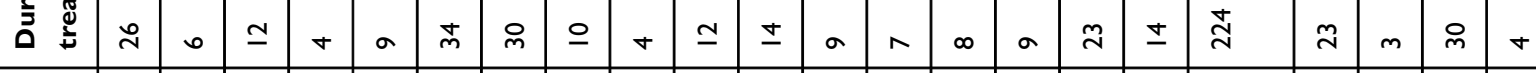

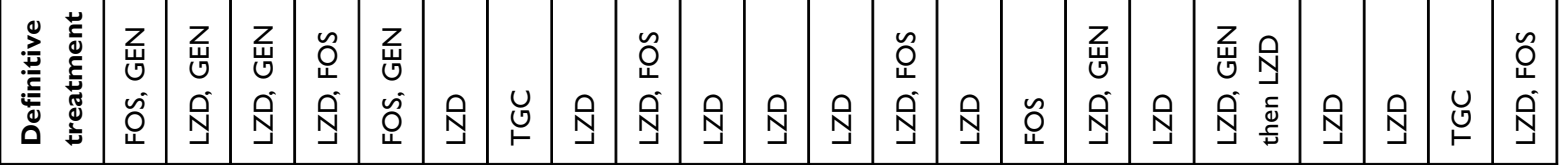

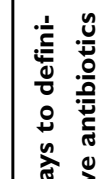

$\overline{\mathrm{u}}$

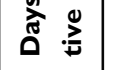

$\sim$

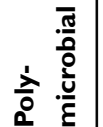

䒕

宽

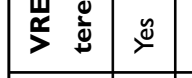

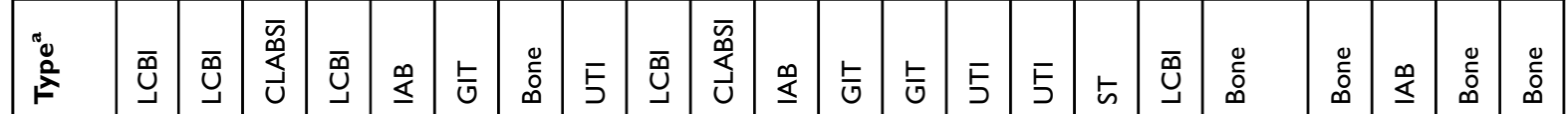

$\stackrel{\square}{\Psi}$

ฮู

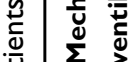

$\overline{\mathrm{u}}$

\begin{tabular}{llllllllllllll|l|l|l|l|l|l|l|l|l|}
$m$ & 0 & & & & & & & & & & & & & & & & & & & & \\
\end{tabular}

m

\begin{tabular}{l|l|l|l|l}
$\circ$ & $\wedge$ & - & $\wedge$ & $\sigma$ \\
\hline
\end{tabular}

\begin{tabular}{llll|l|l|l} 
N & in & in & n & in & \\
\hline & & & & &
\end{tabular}




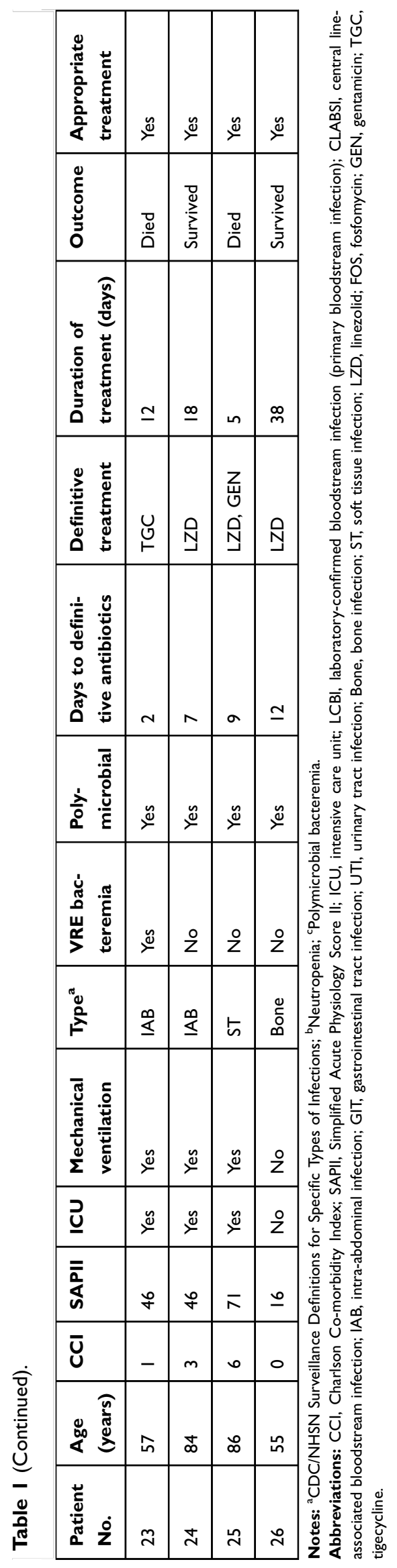

Table 2 Type of infection and definitive treatment of patients infected with vancomycin-resistant enterococci

\begin{tabular}{|l|l|}
\hline Characteristics & N (\%) \\
\hline Type of infection by site & \\
- Gastrointestinal system infection & $8(30.8)$ \\
- Bloodstream infection & $7(26.9)$ \\
- Bone and joint infection & $6(23.1)$ \\
- Ukin and soft tissue infection & $2(7.7)$ \\
\hline VRE bacteremia & $3(11.5)$ \\
\hline Polymicrobial infection & $13(50)$ \\
- Candida spp. & \\
- Enterobacteriaceae & $7(26.9)$ \\
- Pseudomonas aeruginosa & $6(23.1)$ \\
- Acinetobacter baumannii & $2(7.7)$ \\
\hline Days to definitive antibiotics & $2(7.7)$ \\
\hline Definitive monotherapy & $3.5(\mathrm{IQR} 2-7)$ \\
- Linezolid & $15(57.7)$ \\
- Tigecycline & $1 \mathrm{II}(42.3)$ \\
- Fosfomycin & $3(\mathrm{II.5)}$ \\
\hline Definitive combination therapy & $\mathrm{I}(3.8)$ \\
- Linezolid-gentamicin & $1 \mathrm{I}(42.3)$ \\
- Linezolid-fosfomycin & $5(19.2)$ \\
- Fosfomycin-gentamicin & $4(15.4)$ \\
\hline
\end{tabular}

Abbreviation: VRE, vancomycin-resistant enterococci.

were commonly used prior to diagnosis of a VRE infection $(\mathrm{n}=18 ; 69.2 \%$ and $\mathrm{n}=17 ; 65.4 \%$, respectively).

The in-hospital mortality was 19 (73.1\%) and 30-day mortality was 15 (57.7\%) (Table 1). Eighteen patients had microbiological data available after treatment indicating that all had a microbiological cure. The in-hospital mortality for VRE infections by site were 7 (87.5\%) for gastrointestinal, $6(85.7 \%)$ for bloodstream, and $1(16.6 \%)$ for bone and joint infections. Eleven (84.6\%) of 13 patients with VRE bacteremia died. Receiving monotherapy and combination treatments had in-hospital mortality rates of $10(66 \%)$ and $9(81.8 \%)$, respectively. The in-hospital mortality in patients who received a linezolid-based regimen (linezolid alone, linezolid-fosfomycin, or linezolid-gentamicin) was 16 of $20(80.0 \%)$; a fosfomycin-based regimen (fosfomycin alone or fosfomycin-gentamicin), 2 of 3 (66.6\%); and tigecycline alone, 1 of $3(33.3 \%)$. The median SAPII was significantly higher in patients who died compared with those who survived (56 vs $39, P=0.004$ ). AntiVRE regimens, time to definitive therapy, VRE bacteremia, coinfection, age, and comorbidities were not significantly associated with increased in-hospital mortality. Bone and 
joint infection, on the other hand, was significantly associated with a lower in-hospital mortality than other types of infection (OR, 0.022; 95\% CI, 0.002-0.298; $P=0.002$ ). No significant relationships between any clinical characteristics and 30-day mortality were observed.

\section{Molecular typing}

Molecular typing by RAPD was performed for 16 clinical isolates. They were classified in four groups, with 11 strains in group A, 2 in group B, 2 in group C, and 1 in group D (Table 3).

\section{Antimicrobial susceptibility and synergistic effect}

Antimicrobial susceptibility testing was performed in the same 16 strains typed by RAPD (Table 3 ). The MIC range for linezolid was $0.75-2 \mu \mathrm{g} / \mathrm{mL}$, for tigecycline $0.064-0.5$ $\mu \mathrm{g} / \mathrm{mL}$, and for fosfomycin 96 to $>1,024 \mu \mathrm{g} / \mathrm{mL}$. Five $(31.3 \%)$ of the 16 strains were highly resistant (MIC $>500 \mu \mathrm{g} / \mathrm{mL}$ ) to gentamicin.

A synergistic antimicrobial effect was observed for 12 VRE strains. This included $1(8.3 \%)$ for fosfomycin with tigecycline and $3(25 \%)$ for fosfomycin with linezolid. For $9(75 \%)$ strains, there was an additive effect of fosfomycin with linezolid or with tigecycline. A fourfold or more reduction in MIC of linezolid in the presence gentamicin at concentrations of $0.25,0.5,1,2$, and $4 \mu \mathrm{g} / \mathrm{mL}$ was seen in $12.5 \%, 33.3 \%, 58.3 \%, 83.3 \%$, and $91.7 \%$ of strains, respectively. The corresponding results for fosfomycin plus gentamicin were $25 \%, 37.5 \%, 62.5 \%, 62.5 \%$, and $62.5 \%$, respectively (Table 3 ). No antagonism with any combination of antibiotics was observed.

\section{Correlation between in vitro activity, treatment regimens, and treatment outcomes}

Of 26 patients, $2(7.1 \%)$ received inappropriate definitive treatment, 1 with fosfomycin monotherapy and 1 with low dose fosfomycin plus gentamicin, despite high MICs for fosfomycin, $>1,024 \mu \mathrm{g} / \mathrm{mL}$ in 1 and $192 \mu \mathrm{g} / \mathrm{mL}$ in the other. The in-hospital mortality rate among patients given appropriate treatment was $70.8 \%(17 / 24)$. All patients in the inappropriate definitive treatment group were dead (Table 1).

\section{Discussion}

This is the first study worldwide investigating in vitro activity of fosfomycin combined with gentamicin and the first study in Thailand evaluating treatment outcomes for patients with VRE infections. The mortality rates for VRE infections of any type and for VRE bacteremia in particular were higher than in previous studies. ${ }^{16,17}$ This could be attributed to the fact that the patients included in this study were older than those in previous studies. Older patients have multiple and more serious comorbidities and more frequently visit hospital wards. Nonetheless, age and CCI score were not associated with mortality rate, and patients who died were older and had more multiple comorbidities than survivors. The frequency of polymicrobial infections was also observed to be more than that reported in other studies. ${ }^{16-18}$ In this study, polymicrobial infections were not associated with mortality rate probably because of the small sample size. Polymicrobial infections reportedly have a higher mortality rate than monomicrobial infections. ${ }^{19,20}$ More severe illness was significantly associated with high in-hospital mortality. Several other studies have also indicated that a higher severity of illness is associated with greater mortality, including two studies of VRE bacteremia from Taiwan. ${ }^{16,21}$ However, in contrast to our finding that the anti-VRE regimens were not statistically associated with mortality, these two studies reported that the use of linezolid or daptomycin or an antimicrobial with in vitro activity against VRE was associated with lower mortality rate.

A previous study of fosfomycin plus linezolid or tigecycline showed synergism in time-kill studies of approximately $10 \%$ and $30 \%$, respectively. ${ }^{22}$ Combining linezolid with gentamicin improved the activity of linezolid and it exerted bactericidal effect at 72 hrs. ${ }^{23}$ To the best of our knowledge, ours is the first study to focus on the combination of fosfomycin and gentamicin to treat VRE.

In our study, linezolid showed better activity against VRE strains compared with that reported from studies conducted in other countries. ${ }^{24,25}$ VRE strains were susceptible to linezolid with high MICs (MIC50 $1.5 \mu \mathrm{g} / \mathrm{mL}$, MIC90 $2 \mu \mathrm{g} / \mathrm{mL}$ ). In critically ill patients, standard dosing of $600 \mathrm{mg}$ linezolid intravenously twice a day led to subtherapeutic levels. ${ }^{26}$ A Monte Carlo simulation study showed that a standard dose of linezolid can achieve a probability of target attainment of $90 \%$ or more at an MIC $\leq 1 \mu \mathrm{g} / \mathrm{mL}$, but when the MIC was $2 \mu \mathrm{g} / \mathrm{mL}, 600 \mathrm{mg}$ every 8 hrs could provide good clinical efficacy. ${ }^{27}$ However, increasing the dose of linezolid risks greater hematologic toxicity. Linezolid remains one of the treatments of choice, although we suggest that combining it with fosfomycin or gentamicin may be of benefit in critically ill patients with 


\begin{tabular}{|c|c|c|c|c|c|c|c|c|c|}
\hline$\stackrel{9}{9} . \stackrel{\circ}{2}$ & 忞安安 & & $z$ & & & & & & 妾妾 \\
\hline 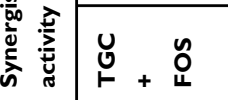 & 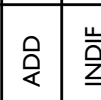 & $\frac{q}{4}$ & \begin{tabular}{l|l}
$z$ \\
$z$
\end{tabular} & 高 & & & $\frac{1}{2}$ & & 㔛 \\
\hline
\end{tabular}

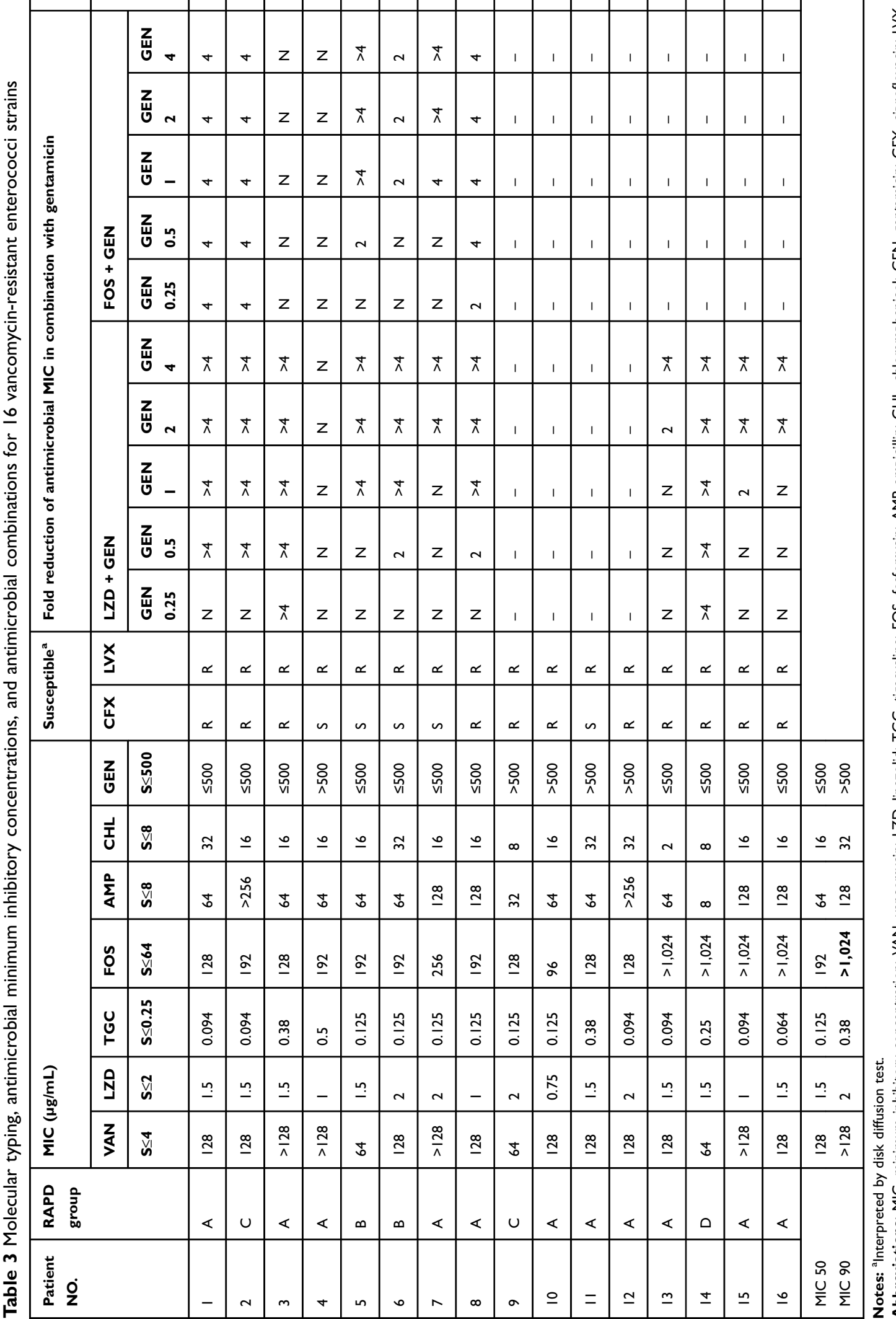


severe infection or if the linezolid MIC is $2 \mu \mathrm{g} / \mathrm{mL}$. The MIC50 and MIC90 of tigecycline was higher than reported from Taiwan $(0.125,0.38 \mu \mathrm{g} / \mathrm{mL}$ vs $\leq 0.03,0.06 \mu \mathrm{g} / \mathrm{mL}){ }^{28}$ Tigecycline remained active against VRE in our patients, but it had a low serum concentration. As VRE bacteremia is common, combining high dose tigecycline with fosfomycin is recommended over monotherapy. Tigecycline monotherapy is more appropriate for skin and soft tissue or intra-abdominal infections.

Fosfomycin is an alternative drug for VRE. In this study, fosfomycin was less active compared with activity shown in previous studies. ${ }^{29,30}$ Use of fosfomycin should be based on MIC, and combination therapy is recommended both for synergistic activity and to prevent resistance. A synergistic effect or adequate reduction of MIC in strains with a fosfomycin MIC $>1,024 \mu \mathrm{g} / \mathrm{mL}$ is difficult. Two patients in our series received subtherapeutic doses. Combining fosfomycin with gentamicin is a potential option to treat VRE, especially for urinary tract infections or when the use of linezolid and tigecycline must be limited. This regimen is recommended when the fosfomycin $\mathrm{MIC}$ is $\leq 128 \mu \mathrm{g} / \mathrm{mL}^{31}$ and may overcome MICs of $256 \mu \mathrm{g} / \mathrm{mL}$ for synergy. The synergistic effect of gentamicin was acceptable for strains not highly resistant to gentamicin. Conventional dosing of gentamicin can be used along with monitoring serum concentrations. The optimal trough concentration to maintain synergism is about $1 \mu \mathrm{g} / \mathrm{mL}$.

In this study, we assumed that strains with DNA differing by one or more bands were unrelated. Pattern A was the predominant pattern, with 3 isolates in 2015, 4 in 2016, and 4 in 2017. These patients had been treated in different wards. There is a potential for spread within the hospital from one ward to another by patients, health care workers, or environmental contamination. Chotiprasitsakul et al reported a VRE outbreak with the same RAPD patterns in 32 isolates. They concluded that contact isolation provides optimal protection against the spread of VRE. ${ }^{32}$

The current study has several limitations. First, this is a retrospective study with a small number of patients which makes it difficult to determine the precise effects of antibiotic regimens and their toxicities. Second, only $16 \mathrm{VRE}$ isolates from 26 patients were available for susceptibility testing, with only 12 strains used to determine synergistic antimicrobial activity. Further studies are required to investigate the bactericidal activity of antimicrobial combinations against VRE.

\section{Conclusion}

The in-hospital mortality rate of VRE infections was high and was particularly associated with more severe illness. Linezolid and tigecycline had good activity, but fosfomycin had less activity against VRE in our institution. Linezolid remains the first-line treatment, but it is worth considering the combination linezolid with gentamicin or fosfomycin to treat severe infection. Fosfomycin with gentamicin is an alternative combination that avoids linezolid toxicity.

\section{Acknowledgments}

Thank you to the Microbiology Laboratory Unit of Phramongkutklao Hospital for VRE strains and all authors for all aspects of the work.

\section{Disclosure}

The authors report no conflicts of interest in this work.

\section{References}

1. Murray BE. Vancomycin-resistant enterococcal infections. $N$ Engl $J$ Med. 2000;342(10):710-721. doi:10.1056/NEJM200003093421007

2. O'Driscoll T, Crank CW. Vancomycin-resistant enterococcal infections: epidemiology, clinical manifestations, and optimal management. Infect Drug Resist. 2015;8:217-230.

3. Chiang H-Y, Perencevich NE, Nair R, et al. Incidence and outcomes associated with infections caused by vancomycin-resistant enterococci in the United States: systematic literature review and metaanalysis. Infect Control Hosp Epidemiol. 2017;38:203-215. doi:10.1017/ice.2016.254

4. Prematunge C, MacDougall C, Johnstone J, et al. VRE and VSE bacteremia outcomes in the era of effective VRE therapy: a systematic review and meta-analysis. Infect Control Hosp Epidemiol. 2016;37:26-35. doi:10.1017/ice.2015.228

5. World Health Organization. WHO Publishes List of Bacteria for Which New Antibiotics are Urgently Needed. WHO Department of Communications; 2017. Available from: https://www.who.int/newsroom/detail/27-02-2017-who-publishes-list-of-bacteria-for-whichnew-antibiotics-are-urgently-needed. Accessed 4 July, 2019.

6. Yim J, Smith JR, Rybak MJ. Role of combination antimicrobial therapy for vancomycin-resistant Enterococcus faecium infections: review of the current evidence. Pharmacotherapy. 2017;37:579592. doi: $10.1002 /$ phar. 1922

7. National Antimicrobial Resistance Surveillance Center. Thailand [homepage on the internet]. Antimicrobial Resistance 2000-2016. Available from: http://narst.dmsc.moph.go.th/. Accessed February 3, 2019.

8. Thongkoom P, Kanjanahareutai S, Chantrakooptungool S, Rahule S. Vancomycin-resistant enterococci (VRE) isolates isolated in Rajavithi Hospital between 1999 and 2009. J Med Assoc Thai. 2012;95(Suppl 3):S7-15.

9. Center for Disease Control and Prevention [homepage on the internet]. CDC/NHSN Surveillance Definitions for Specific Types of Infections; 2019. Available from: https://www.cdc.gov/nhsn/pdfs/pscmanual/ 17pscnosinfdef_current. Accessed February 3, 2019.

10. Barbier N, Saulnier P, Chachaty E, Dumontier S, Andremont A. Random amplified polymorphic DNA typing versus pulsed-field gel electrophoresis for epidemiological typing of vancomycin-resistant enterococci. J Clin Microbiol. 1996;34:1096-1099. 
11. Tenover FC, Arbeit RD, Goering RV, et al. Interpreting chromosomal DNA restriction patterns produced by pulsed-field gel electrophoresis: criteria for bacterial strain typing. J Clin Microbiol. 1995;33:22332239

12. Clinical and Laboratory Standard Institute. Performance Standards for Antimicrobial Susceptibility Testing: Twenty-Eighth Informational Supplement. CLSI Document M100. Wayne (PA): Clinical and Laboratory Standard Institute; 2018.

13. The European Committee on Antimicrobial Susceptibility Testing [homepage on the internet]. Breakpoint tables for interpretation of MICs and zone diameters; 2018. Available from: http://www.eucast. org/clinical_breakpoints/. Accessed December 23, 2018.

14. Charlson ME, Pompei P, Ales KL, MacKenzie CR. A new method of classifying prognostic comorbidity in longitudinal studies: development and validation. J Chronic Dis. 1987;40:373-383.

15. Beck DH, Smith GB, Pappachan JV, Millar B. External validation of the SAPS II, APACHE II and APACHE III prognostic models in South England: a multicentre study. Intensive Care Med. 2003;29:249-256. doi:10.1007/s00134-002-1607-9

16. Ye JJ, Shie SS, Cheng CW, et al. Clinical characteristics and treatment outcomes of vancomycin-resistant Enterococcus faecium bacteremia. $J$ Microbiol Immunol Infect. 2018;51:705-716. doi:10.1016/j. jmii.2017.08.025

17. Jiang H-L, Zhou Z, Wang L-S, Fang Y, Li Y-H, Chu C-I. The risk factors, costs, and survival analysis of invasive VRE infections at a Medical Center in Eastern Taiwan. Int J Infect Dis. 2017;54:18-24. doi:10.1016/j.ijid.2016.11.005

18. Cheah AAY, Spelman T, Liew D, et al. Enterococcal bacteraemia: factors influencing mortality, length of stay and costs of hospitalization. Clin Microbiol Infect. 2013;19:181-189. doi:10.1111/14690691.12132

19. Lin JN, Lai C-H, Chen YH, et al. Characteristics and outcomes of polymicrobial bloodstream infections in the emergency department: a matched case-control study. Acad Emerg Med. 2010;17:1072-1079. doi:10.1111/j.1553-2712.2010.00871.x

20. McKenzie FE. Case mortality in polymicrobial bloodstream infections. $J$ Clin Epidemiol. 2006;59:760-761. doi:10.1016/j. jclinepi.2005.12.009

21. Chou CH, Lee NY, Lee HC, Chang CM, Lee CC, Ko WC. Emergence of vancomycin-resistant enterococcus bloodstream infections in southern Taiwan. J Microbiol Immunol Infect. 2012;45:221227. doi:10.1016/j.jmii.2011.11.005

22. Tang HJ, Chen CC, Zhang CC, et al. In vitro efficacy of fosfomycinbased combinations against clinical vancomycin-resistant Enterococcus isolates. Diagn Microbiol Infect Dis. 2013;77:254257. doi:10.1016/j.diagmicrobio.2013.07.012
23. Luther MK, Arvanitis M, Mylonakis E, LaPlante KL. Activity of daptomycin or linezolid in combination with rifampin or gentamicin against biofilm-forming Enterococcus faecalis or E. faecium in an in vitro pharmacodynamic model using simulated endocardial vegetations and an in vivo survival assay using Galleria mellonella larvae. Antimicrob Agents Chemother. 2014;58:4612-4620. doi:10.1128/AAC.02790-13

24. Yadav G, Thakuria B, Madan M, Agwan V, Pandey A. Linezolid and vancomycin resistant enterococci: a therapeutic problem. J Clin and Diagn Res. 2017;11:GC07-GC11.

25. de Almeida LM, de Araújo MRE, Iwasaki MF, et al. Linezolid resistance in vancomycin-resistant Enterococcus faecalis and Enterococcus faecium isolates in a Brazilian hospital. Antimicrob Agents Chemother. 2014;58:2993-2994. doi:10.1128/AAC.02399-14

26. Zoller M, Maier B, Hornuss C, et al. Variability of linezolid concentrations after standard dosing in critically ill patients: a prospective observational study. Crit Care. 2014;18:R148. doi:10.1186/cc13984

27. Yang M, Zhang J, Chen Y, et al. Optimization of linezolid treatment regimens for Gram-positive bacterial infections based on pharmacokinetic/pharmacodynamic analysis. Future Microbiol. 2017;12:3950. doi:10.2217/fmb-2016-0140

28. Tsai HY, Liao $\mathrm{CH}$, Chen $\mathrm{YH}$, et al. Trends in susceptibility of vancomycin-resistant Enterococcus faecium to tigecycline, daptomycin, and linezolid and molecular epidemiology of the isolates: results from the Tigecycline In Vitro Surveillance in Taiwan (TIST) study, 2006 to 2010. Antimicrob Agents Chemother. 2012;56:3402. doi:10.1128/AAC.00533-12

29. Lu CL, Liu CY, Huang YT, et al. Antimicrobial susceptibilities of commonly encountered bacterial isolates to fosfomycin determined by agar dilution and disk diffusion methods. Antimicrob Agents Chemother. 2011;55:4295-4301. doi:10.1128/AAC.00349-11

30. Falagas ME, Roussos N, Gkegkes ID, Rafailidis PI, Karageorgopoulos DE. Fosfomycin for the treatment of infections caused by Gram-positive cocci with advanced antimicrobial drug resistance: a review of microbiological, animal and clinical studies. Expert Opin Investig Drugs. 2009;18:921-944. doi:10.1517/ 13543780902967624

31. Asuphon O, Montakantikul P, Houngsaitong J, Kiratisin P, Sonthisombat P. Optimizing intravenous fosfomycin dosing in combination with carbapenems for treatment of Pseudomonas aeruginosa infections in critically ill patients based on pharmacokinetic/pharmacodynamic (PK/PD) simulation. Int J Infect Dis. 2016;50:23-29. doi:10.1016/j.ijid.2016.06.017

32. Chotiprasitsakul D, Santanirand P, Thitichai P, et al. Epidemiology and Control of the first reported vancomycin-resistant enterococcus outbreak at a tertiary-care Hospital in Bangkok, Thailand. Southeast Asian J Trop Med Public Health. 2016;47:494-502.
Infection and Drug Resistance

\section{Publish your work in this journal}

Infection and Drug Resistance is an international, peer-reviewed openaccess journal that focuses on the optimal treatment of infection (bacterial, fungal and viral) and the development and institution of preventive strategies to minimize the development and spread of resistance. The journal is specifically concerned with the epidemiology of antibiotic resistance and the mechanisms of resistance development and diffusion in both hospitals and the community. The manuscript management system is completely online and includes a very quick and fair peerreview system, which is all easy to use. Visit http://www.dovepress.com/ testimonials.php to read real quotes from published authors. 